\title{
Theoretical aspects of monitoring synergistic processes in the rock massif
}

\author{
Tetiana Palamarchuk ${ }^{1, *}$ \\ ${ }^{1}$ Institute of Geotechnical Mechanics named by N. Poljakov of National Academy of Sciences of \\ Ukraine, 49005, Dnipro, Simferopolska Str., 2a, Ukraine
}

\begin{abstract}
In this article, the grounds for considering of rocks massif as a nonlinear medium with dissipative properties have been shown that as a result of the combined influence of normal and tangential stresses around the workings, synergistic effects are observed with the formation of similar structures, which leads to the appearance of a quasi-stationary damped stress wave, and this, in turn, makes it possible to predict the nature of changes in the stress-strain state of the rock massif in space-time continuum. Taking into account the influence of the mining operations speed, a theoretical justification for diagnostics the physicomechanical properties and the stress state of sedimentary rocks has been developed by using the dynamic and kinematic characteristics of acoustic and electric fields. The theoretically established relationship of the stress state of massif of quartz-containing rocks with its elastic and electrical parameters follows taking into account the damped wave nature of the processes around the mine workings. Obtained results indicates the principal possibility of quantitative assessment of stress components acting in the rock massif using determined physical parameters of the massif.
\end{abstract}

\section{Introduction}

Rocks, geological sections are characterized by a high degree of heterogeneity, the presence of large and small blocks, and various fracture systems. Efficiency and safety of operation of underground and surface structures substantially depend on the level of our knowledge about the geomechanical state of their environment and about the regularity by which various physical processes take place in the rock massif, how synergistic and structural phase transitions occur in natural and man-made systems. The speed and intensity of these processes determine the speed and intensity of underground structure's deformation. At the same time, the range of deformation can fluctuate from elusive changes to instantaneous gas-dynamic phenomena (emissions, rock bursts, etc.), as well as earthquakes.

One of the specific features of geosystems is their ability to self-organize (selfconstruct) under the influence of the energy of the processes occurring on the Earth [1 - 3]. The question of the self-organization of open natural systems, covering living and inanimate nature, including geosystems, was raised by the second law of thermodynamics.

\footnotetext{
*Corresponding author: tp208_2008@ukr.net
} 
In [4], the process of a fracture development zone around a borehole drilled in clay rocks was investigated, and the walls of this borehole were strengthened by applying a polymer coating. The authors established a complex sequence of cracks development around the borehole and proved the important role of pore pressure, which gradually dissipated as the fracture zone developed. However, from the standpoint of the possible occurrence of dissipative structures around the borehole, this process has not been studied and remained outside the attention of researchers.

D. Sahar and co-workers investigated the problem of determining stress components in a rock massif using the parameters of the so-called break-out or fracture zone around the borehole. This method is widely used to restore the stress's components acting in a rock massif to the start of mining works. The authors investigated the stress-strain state around the borehole, which was destroyed due to exceeding the tensile strength of the massif surrounding this borehole. The authors found that the depth at which the break-out began, and its value are dependence on the stress in the massif surrounding the borehole [5].

In [6], an interesting phenomenon of rock massif destruction spread far from the front mining work. They called this phenomenon a domino effect, which contributes to the spontaneous maintenance of cracks growth under the condition that adjacent parts of the massif are in contact with the boundary state. In fact, the authors confirmed the possibility of the process of self-organization emergence during the irreversible deformation of the rock massif and identified another form of dissipative structures of strength loss in the form of a chain effect.

In the literature there are a large number of such experiments descriptions. However, their common flaw repeats what was formulated in the previous analysis: almost all publications are limited only to the identification of dissipative structures and do not determine their evolution. From the kinematics standpoint of dissipative structures, such data adds little information. However, it is obvious that dissipative structures in space and in time appear unevenly. There is also a large spatial variation in the places of occurrence of microseismic activity, which indicates the random nature of dissipative structures evolution.

The study of the rock massif structures as a form of self-organization of the geophysical environment will provide the most objective information about the properties and state of the rock massif, geomechanical systems and the stability of underground structures.

\section{Methods}

To solve this problem, we used methods of continuum mechanics, rock mechanics, mathematical physics, empirical and analytical methods, as well as analysis and synthesis of results. The reliability and validity of the results obtained is confirmed by the use of proven theoretical research methods, as well as by taking into account the results of experimental studies.

\section{Results and discussion}

Taking into account the results obtained when considering the processes occurring in the vicinity of the mining working of a circular section [2] in this article, taking into account the speed of mine working operations and experimental data, the following expression was obtained:

$$
\sigma=\sigma_{0}(0.11 \ln V+1) \exp \left(-\left(\alpha r+\frac{t}{\tau}\right)\right) \cos \frac{\pi}{2 R_{0}} r
$$


where $\sigma_{0}$ - the initial stresses on the mining working contour; $V$ - the speed of mining work; $r$ - the distance from the mining working contour to the deep into the massif; $\alpha$ - the attenuation coefficient of a quasi-stationary wave; $t$ - time, $\mathrm{s} ; \tau-$ the relaxation time, $\mathrm{s} ; R_{0}-$ working radius, $\mathrm{m}$.

Similar processes are also observed not only in the radial direction $r$ around the mining working, but also along its generatrix $L$, therefore the change in stresses along mining workings is described by law:

$$
\sigma=\sigma_{0}(0.11 \ln V+1) \exp \left(-\left(\alpha L+\frac{t}{\tau}\right)\right) \cos \frac{\pi}{2 R_{0}} L .
$$

Thus, the effect of compression and tension zone displacement along the generatrix of extended underground objects, which can be defined as quasi-wave, is substantiated.

A mechanism is proposed to explain the known wave nature of changes in rock pressure around disturbances in the rock massif and self-organization processes that occur in it, leading to a steady state as a result of self-destruction of macroscopic nonlinear dissipative systems, which include geomechanical systems. This mechanism is that as a result of exposure geological and technogenic factors the massif goes from one quasi-stationary equilibrium state with the minimum potential energy to another.

For a nonequilibrium state of a system for which an inequality holds $d S / d t \geq 0$, its entropy can be defined as follows:

$$
S=-k H+S_{0},
$$

where $k$ - the Boltzmann constant; $S_{0}=k \cdot \ln \omega$; $\omega$ - probability density function; $H$ Boltzmann function, equal to

$$
H(t)=\int_{(\mathbf{r})(\mathbf{v})} f(\mathbf{r}, \mathbf{V}, t) \ln (\mathbf{r}, \mathbf{V}, t) d \mathbf{r} d \mathbf{V}
$$

$f(\mathbf{r}, \mathbf{V}, t)$ - distribution function over coordinates $\mathbf{r}$, velocities $\mathbf{V}$ and time $t$, and for stationary states $d H / d t=0$, and for non-stationary $d H / d t<0$.

The entropy thus determined obeys the law of entropy increase.

If the increase in entropy characterizes the growth of chaos in the system, then the selforganization of the system is characterized a decrease in entropy.

Those, entropy is one of the most important parameters characterizing the processes of the system self-organization. The parameters characterizing the degree of the system selforganization can also include:

- the fragility of rocks

$$
\psi=\frac{\sigma_{p}}{\sigma_{c}}
$$

where $\sigma_{p}$ - tensile strength of rocks; $\sigma_{c}$ - compression strength of rocks;

- coefficient of loosening

$$
k_{p}=1+\frac{\lg \rho^{*}-\lg \rho_{1}^{*}}{\lg \rho_{2}^{*}},
$$


where $\rho^{*}$ - the measured electrical resistivity; $\rho_{1}^{*}-$ electrical resistivity of the undisturbed massif; $\rho_{2}{ }_{2}$ - electrical resistivity of the broken massif;

- coefficient of structural weakening [7]

$$
k_{c}=[1-\sqrt{0.5 \eta} \exp (-0.25 \eta)] \frac{\eta_{0}^{2}+1}{\eta^{2}+1},
$$

where $\eta$ - coefficient of variation of rock massif strength, determined by the formula

$$
\eta=\sqrt{\frac{l_{\mathrm{t}}+l_{0}}{l_{\mathrm{t}}}\left(\eta_{0}^{2}+1\right)-1},
$$

where $l_{\mathrm{t}}$ - the average distance between cracks; $l_{0}-$ the characteristic size of the standard rock sample; $\eta_{0}$ - coefficient of variation laboratory tests of rock samples results.

The effectiveness of technological solutions and the rhythm of equipment operation depend on the degree of knowledge of the geological conditions, stress-strain state, properties of the massif, structural heterogeneities, such as the boundary between rocks, tectonic disturbances in the formations, karst, voids and cracks. One of the decisive conditions for reducing the cost of mining and improving the safety of work is the timely monitoring of geotechnical structures stability, properties and stress-strain state of the rock massif, which from the point of view of efficiency and informativity should be based on express methods of mining geophysics.

In order to assess the degree of informativeness dynamic and kinematic characteristics of acoustic and electromagnetic fields for diagnosing the stress state of a rock massif, is considered sedimentary rocks that can be considered dielectrics. As is known, under normal conditions, the dielectric is electrically neutral, i.e. contains the same number of positive and negative charges. The introduction of a dielectric into an electric field strength changes this field. The electric field vector $\mathbf{E}$ and the induction vector $\mathbf{D}$ will no longer be parallel, and their difference will give a polarization vector $\mathbf{P}$.

As a result of solving the system of equations describing the propagation of acoustic waves in seismoelectrically active layered rocks, taking into account the initial stress state, we obtain in the first approximation an expression for the velocities and attenuation coefficients of longitudinal waves and electric field strength:

$$
\begin{gathered}
V_{3 p}=\left(\frac{\lambda_{1}\left(1 \pm \sigma_{33}^{0} / 3 K\right)}{\rho}\right)^{1 / 2}\left(1-\frac{\omega^{2} \eta_{1} \tau_{1}}{2 \lambda_{1}\left(1+\omega^{2} \tau_{1}^{2}\right)}-\frac{d_{1}^{2}}{\lambda_{1} b_{1}}\right), \\
\alpha_{3 p}=\left(\frac{\rho}{\lambda_{1}\left(1 \pm \sigma_{33} / 3 K\right)}\right)^{1 / 2} \frac{\omega^{2} \eta_{1}}{2 \lambda_{1}\left(1+\omega^{2} \tau_{1}^{2}\right)}, \\
E_{3}^{L}=-a_{1} P_{1}=-\frac{a_{1} d_{1}\left(1 \pm \sigma_{33}^{0} / 3 K\right)}{b_{1}}
\end{gathered}
$$

for shear waves 


$$
\begin{gathered}
V_{i s}=\left(\frac{4 \lambda_{5}\left(1 \pm \sigma_{i i}^{0} / 3 K\right)}{\rho}\right)^{1 / 2}\left(1-\frac{\omega^{2} \eta_{5} \tau_{5}}{2 \lambda_{5}\left(1 \pm \sigma_{i i}^{0} 3 K\right)\left(1+\omega^{2} \tau_{5}^{2}\right)},(i=1,2)\right. \\
\left.-\frac{2 d_{5}\left(2 d_{2}+d_{3}\right)}{\lambda_{5} b_{4}}+\frac{\sigma_{33}^{0}-\sigma_{i i}^{0}}{16 \lambda_{5}\left(1 \pm \sigma_{i i}^{0} / 3 K\right)}\right) \\
\alpha_{i s}=\left(\frac{\rho}{4 \lambda_{5}\left(1 \pm \sigma_{i i} / 3 K\right)}\right)^{1 / 2} \frac{\omega^{2} \eta_{5}}{2 \lambda_{5}\left(1+\omega^{2} \tau_{5}^{2}\right)},
\end{gathered}
$$

where $\lambda_{\mathrm{i}}$ - the elastic parameters of the medium, $\mathrm{Pa} ; K$ - compression module, $\mathrm{Pa} ; \sigma_{i j}^{0}-$ initial stresses perpendicular and parallel to stratification, $\mathrm{Pa} ; \rho-$ density of rocks, $\mathrm{kg} / \mathrm{m}^{3} ; \omega$ - cyclic frequency of acoustic oscillations, $\mathrm{s}^{-1} ; \eta_{\mathrm{i}}$ - viscosity coefficients, $\mathrm{Pa} \cdot \mathrm{s} ; \tau_{\mathrm{i}}-$ the relaxation time, s; $a, b, d$-electric constants; $\mathbf{P}$ - polarization vector, $\mathrm{C} / \mathrm{m}^{2}$.

The velocities of longitudinal and transverse waves depend on the elastic, electrical and piezoelectric properties of rocks, and the dependence on the stressed state remains the same as in rocks that are not modified by an electric field.

The experimental values of the energy density of acoustic and electromagnetic waves can be determined from the following expressions

$$
\begin{gathered}
W_{E}=\sum_{i=1}^{n}\left(A_{i}^{E}\right)^{2} T_{i}^{E} ; \\
W_{u}=\sum_{i=1}^{n}\left(A_{i}^{u}\right)^{2} T_{i}^{u},
\end{gathered}
$$

where $A_{i}^{u, E} ; T_{i}^{u, E}$ - respectively, the amplitude and duration of acoustic and electromagnetic pulses for longitudinal waves.

Taking into account the expressions for the bulk density of acoustic waves

$$
W_{u}=\rho V^{2} \varepsilon^{* 2}=\rho u_{0}^{2} \omega^{2},
$$

and bulk density of electromagnetic waves

$$
W_{E}=\varepsilon \varepsilon_{0} E^{2},
$$

will get

$$
\frac{W_{E}}{W_{u}}=\frac{\varepsilon \varepsilon_{0} a_{1}^{2} d_{1}^{2}\left(1 \pm \sigma_{33}^{0} / K\right)^{2}}{\rho b_{1}^{2} \omega^{2}} .
$$

From here, after simple transformations, taking into account the wave nature of geomechanical processes around the mining working, we obtain the expression

$$
\sigma_{33}^{0}=3 K\left(\frac{W_{E}}{W_{u}} \frac{\lambda_{1} b_{1}^{2} \varepsilon^{* 2}}{a_{1}^{2} d_{1}^{2} \varepsilon \varepsilon_{0}} \mp 1\right) \exp \left(-\left(\alpha r+\frac{t}{\tau}\right)\right) \cos \frac{\pi}{2 R_{0}} r,
$$

from which the theoretically established relationship of the stress state of massif of quartzcontaining rocks with its elastic and electrical parameters follows, which indicates the 
principal possibility of quantitative assessment of stress components acting in the rock massif using determined physical parameters of the massif.

Similarly, the remaining components of the main stresses can be determined.

In the formulas obtained, it is also possible to take into account the speed of mining work, replacing strength and elastic parameters with expressions of the form:

$$
X_{i}=X_{0}(0.11 \ln V+1)
$$

where $X_{0}$ - the strength or elastic parameters of rocks.

The dependence of stresses on the mining works velocity at various depths is given in Table 1. From the table it follows that with increasing depth and mining works velocity operations within the specified limits, the stresses increase.

Table 1. The dependence of stresses on the mining works velocity at various depths.

\begin{tabular}{|c|c|c|c|c|c|}
\hline \multirow{2}{*}{$\begin{array}{c}\text { The mining } \\
\text { works } \\
\text { velocity } V \\
\mathrm{~m} / \mathrm{s}\end{array}$} & \multicolumn{5}{|c|}{ Depth of mining working $H, \mathrm{~m}$} \\
\cline { 2 - 6 } & 300 & 500 & 600 & 700 & 800 \\
\hline 3 & 1.1 & 166.5 & 194.25 & 222.0 & 277.5 \\
\hline 4 & 1.39 & 170.85 & 199.32 & 227.8 & 284.75 \\
\hline 5 & 1.6 & 174.0 & 203.0 & 232.0 & 290.0 \\
\hline 6 & 1.8 & 177.0 & 206.5 & 236.0 & 295.0 \\
\hline 7 & 1.94 & 179.1 & 208.95 & 238.8 & 298.5 \\
\hline
\end{tabular}

\section{Conclusions}

The grounds for considering of rocks massif as a nonlinear medium with dissipative properties have been shown that as a result of the combined influence of normal and tangential stresses around the workings, synergistic effects are observed with the formation of similar structures, which leads to the appearance of a quasi-stationary damped stress wave, and this, in turn, makes it possible to predict the nature of changes in the stress-strain state of the rock massif in space-time continuum.

Taking into account the influence of the mining operations speed, a theoretical justification for diagnostics the physicomechanical properties and the stress state of sedimentary rocks has been developed by using the dynamic and kinematic characteristics of acoustic and electric fields. The theoretically established relationship of the stress state of massif of quartz-containing rocks with its elastic and electrical parameters follows taking into account the damped wave nature of the processes around the mine workings. Obtained results indicate the principal possibility of quantitative assessment of stress components acting in the rock massif using determined physical parameters of the massif.

\section{References}

1. Pashkin, Ye.M., (1992). Synergetics of geosystems: a new approach in engineering geology. Ingenernaya geologiya, (6), 11-13

2. Palamarchuk, T.A., Kirichenko, V.Ya., Usachenko, B.M., (2006). Elementy mekhanosinergetiki porodnogo massiva. Dnepropetrovsk: Lira LTD

3. Zakharova, L.M., (2016). Fizychni osnovy evoliutsii dysypatyvnykh struktur v geomekhanitsi. Saarbruken: Lambert Academic Publishing 
4. C.S. Villy, A. Kontogianni, International Journal of Rock Mechanics and Mining Sciences, 46 (1), 182-187 (2009)

5. D. I. Sahar, M. Schoenball, E. Gerolymaton, T. Kohl, International Journal of Rock Mechanics and Mining Sciences, 97, 134-143 (2017)

6. D. Wallis, G.E. Lloyd, R.J. Phillips, A.J. Parsons, R.D. Walshaw, Journal of Structural Geology, 77, 45-61 (2015)

7. Shashenko, A.N., (2001). Mekhanika gornykh porod. Dnepropetrovsk: NGAU 